\title{
Kontinuität oder Wandlung? \\ Stadt- und Landesplanung am Geographischen Institut der Universität Wien um 1938
}

petra.svatek@univie.ac.at, Österreichische Akademie der Wissenschaften

eingereicht am: 01.07.2018, akzeptiert am: 20.09.2018

Dieser Artikel setzt sich mit der Stadt- und Landesplanung am Geographischen Institut der Universität Wien in den 1930er und 1940er Jahren auseinander und hinterfragt die Kontinuitäten und Wandlungen, welche die Eingliederung Österreichs in das nationalsozialistische Deutsche Reich mit sich gebracht hat. Bereits vor 1938 zeichnete sich in der Forschung das rassistische Konzept des völkischen Nationalismus ab. Im Jahr 1938 kam es schließlich zu einer Intensivierung von Tendenzen, die bereits zuvor vorhanden waren, aber auch zu neuen Verbindungen zur Politik und zu neuen Forschungsthemen.

Keywords: Geographisches Institut Universität Wien, Nationalsozialismus, Wissenschaft und Politik, Raumforschung

\section{Continuity or change? Urban and regional planning at the Department of Geography of the University of Vienna around 1938}

This article deals with urban and regional planning at the Department of Geography at the University of Vienna in the 1930s and 1940s. It questions the continuities and changes which the integration of Austria into the National Socialist German Reich entailed. Even before 1938, racist intentions in research were partly recognizable. In 1938, there was an intensification of such tendencies, which had already existed before; however, also new connections to politics and new research topics could be found.

Keywords: Department of Geography at the University of Vienna, National Socialism, Science and Politics, Spatial Research

\section{Einführung}

Im Jahr 2018 jährt sich der Anschluss Österreichs an das Deutsche Reich zum 80. Mal. Vor allem in den letzten beiden Jahrzehnten hat in Österreich in fast allen Wissenschaftsdisziplinen eine intensive Aufarbeitung der NS-Zeit begonnen. In dieser Arbeit konnte festgestellt werden, dass die Geographie diesem Forschungstrend eindeutig hinterherhinkt. Doch die Erforschung der Geschichte des eigenen Faches ist heute auch für Geographielehrerinnen und -lehrer und zudem für den Schulunterricht im Bereich der politischen Bildung von Bedeutung (vgl. Sitte 2014). Der Artikel soll außerdem einen impliziten Beitrag zur „kritischen Art der Geschichtsschreibung“ im Fachgebiet Geographie liefern, bei der nicht immer von „einem positiven Verhältnis zur Vergangenheit“ ausgegangen wird und ,außer- und überfachliche
Zusammenhänge analysiert" (Wardenga 1995: 523, 525) werden. Am 12. März 1938 erfolgte die Eingliederung Österreichs in das nationalsozialistische Deutsche Reich. Doch wie ist dieses Ereignis für die Stadt- und Landesplanung, die damals bereits als Teil des Wissensgebietes Raumforschung galt, am Geographischen Institut der Universität Wien zu bewerten?

Die Raumforschung stand erst am Beginn, ein eigenes Wissensgebiet zu werden. Sowohl ihr Institutionalisierungsprozess als auch ihre methodische Entwicklung standen noch am Anfang. Das Forschungsfeld, was damals unter Raumforschung verstanden wurde, war noch nicht klar definiert. Humangeographische Aspekte wurden nämlich in der deutschsprachigen Geographie bis zum Ersten Weltkrieg, bis auf einige Ausnahmen (Bsp. Friedrich Ratzel), zugunsten einer physiogeographischen Orientierung weitgehend vernachlässigt. Erst die drohende Niederlage im Krieg 
und die Pariser Friedensverhandlungen veranlassten auch die Geographen, sich verstärkt mit der zeitgenössischen Geographie des Menschen und mit angewandten landeskundlichen Forschungen auseinanderzusetzen. Zeitgleich intensivierte sich auch die Verbindung zur Politik, die ab der NS-Zeit schließlich eine Verstärkung erfahren hatte. Dadurch wurden für die Geographen Themen wichtig, die sonst wahrscheinlich nicht in ihrem Themenrepertoire zu finden gewesen wären. (vgl. Wardenga et. al. 2011)

Zeitgleich erhielt auch die Kartographie eine immense Aufwertung, da sie für dieses neue Wissensgebiet wichtige Ressourcen zur Verfügung stellen konnte. Diese Beziehung beruhte allerdings auf Gegenseitigkeit, da die Kartographie auch von der Raumforschung beeinflusst wurde, zum Beispiel im Bereich der Methodenentwicklung und in der leicht veränderten Forschungspraxis. Wie der deutsche Geograph Walter Geisler (1891-1945) erklärte, stellt die Raumforschung „die Kartographie vor neuen Aufgaben, und die Kartographie ermöglicht es der Raumforschung erst, Aufgaben nicht nur darzustellen, sondern auch durch die Darstellung zu erkennen. Raumforschung und Kartographie befruchten sich wechselseitig und müssen Hand in Hand arbeiten" (Geisler 1942: 43). Auch am Geographischen Institut der Universität Wien erhielt die Kartographie ab den frühen 1930er Jahren mit dem Burgenlandatlas und in weiterer Folge mit dem Gauatlas Niederdonau einen enormen Aufschwung.

Die Institutionalisierung der Raumforschung begann im Deutschen Reich Ende des Jahres 1935, als die Reichsarbeitsgemeinschaft für Raumforschung (RAG) von Bernhard Rust (Reichsminister für Wissenschaft, Erziehung und Volksbildung) und Hanns Kerrl (Leiter der Reichsstelle für Raumordnung) gegründet wurde. Die von der RAG in den darauffolgenden Monaten und Jahren an deutschen Universitäten und Hochschulen gegründeten Arbeitsgemeinschaften, sollten raumorientierte Forschungen der eigenen Region und angrenzender Gebiete im Sinne der nationalsozialistischen Expansions- und ,Lebensraumpolitik' durchführen. Zum ersten Leiter wurde der Agrarwissenschaftler Konrad Meyer (1901-1973) ernannt, welcher 1939 vom Juristen Paul Ritterbusch (1900-1945) abgelöst wurde. (vgl. Leendertz 2017) Der Begriff „Lebensraum“ war ein „NS-Schlagwort zur Legitimation einer rassisch begründeten gewaltsamen Expansion des Deutschen Reiches" (SchmitzBerning 2007: 375).

Raumforschung wurde damals als eine "Aufgabe“ angesehen, „den deutschen Gesamtraum als den tragenden Grund des deutschen Volkes in seiner landschaftlichen Aufgliederung und seiner unlösbaren Verbindung mit Leben und Leistung dieses Volkes so $\mathrm{zu}$ erforschen und $\mathrm{zu}$ werten, daß diese vertiefte Kenntnis der Kräfte im Raum für den nationalsozialistischen Neubau des Reiches eine sichere und brauchbare Unterlage bildet" (Meyer 1937a: 36). Raumforschung wurde somit explizit als politische Aufgabe formuliert, die nicht nur beschreibend tätig sein, sondern für die Ziele des nationalsozialistischen Regimes auch ein Zukunftsbild zeigen sollte. Obwohl immer wieder der interdisziplinäre Charakter der Raumforschung betont wurde („über Enge und Hemmungen der Fachdisziplinen und Institutsinteressen hinausstreben“, Meyer 1937b: 3), galt die Geographie damals bereits als die wichtigste Raumwissenschaft (vgl. Dörries 1937 \& Rössler 1987). Aus diesem Grund ist ab den 1930er Jahren auch ein enormer Bedeutungsgewinn der Geographischen Institute diverser deutscher Universitäten mit einem gleichzeitigen „Politisierungsprozess“ (Böhm 2008: 373) der geographischen Forschungen erkennbar (vgl. Wardenga 2006; Schultz 2010; Baas 2015). Diese Arbeit geht der Frage nach, ob es so eine Politisierung der Forschungen auch am Geographischen Institut der Universität Wien gab, und welche Kontinuitäten und Wandlungen diese um 1938 erfahren haben.

\section{Die Anfänge der Raumforschung am Geographischen Institut}

Obwohl das Wort ,Raumforschung' im Sprachgebrauch österreichischer Geographen vor 1938 nicht gebräuchlich war, sind am Geographischen Institut der Universität Wien die ersten Studien zur Raumforschung bereits in den 1910er Jahren zu finden. Hugo Hassinger, der am 8. November 1877 in Wien als Sohn eines Beamten geboren wurde und von 1896 bis 1902 an der Universität Wien Geographie und Geschichte studierte, verfasste 1910 zwei Artikel, die sich mit „Aufgaben der Städtekunde“ und der „Siedlungs- und Verkehrsgeographie von Wien“" auseinandersetzen. Hassinger schrieb zu dieser Zeit gerade seine Habilitation über „Die Mährische Pforte und ihre benachbarten Landschaften", in der die Geomorphologie noch einen recht breiten Raum umfasste. Diese beiden Artikel können daher als Beginn seiner stadtplanerischen Forschungen angesehen werden. In den Folgejahren eruierte Hassinger im Kontext der damals bereits immer bedeutender werdenden Denkmal- und Heimatschutzbewegung die Stilepochen aller Wiener Häuser, um für die von der damaligen Politik angeordneten Umgestaltung des alten Stadtbildes ein „Instrument der Baupolitik“ (UAW, NL Hassinger Kt. 27) zur Verfügung zu haben, die „im Kampf um unser altes Wien als Waffe" (Hassinger 1912: 35) verwendet werden sollte. Von 1910 bis 1912 kartierte er alle Bauten 
Wiens vom Mittelalter bis in die 1840er Jahre. Damit führt er an der Universität Wien einen neuen methodischen Ansatz ein, der heute als „morphogenetische Stadtgeographie" (Heineberg 2006: 16) bezeichnet wird. Als Resultat seiner Forschungen entstand 1916 ein „Kunsthistorischer Atlas der k. k. Reichshauptund Residenzstadt Wien und Verzeichnis der erhaltenswerten historischen Kunst- und Naturdenkmale des Wiener Stadtbildes" mit 19 Plänen und 304 Seiten Text. (vgl. Svatek 2015)

\section{Die Periode von 1930 bis 1937}

Hassingers Berufung zum Professor an die Universität Basel beendeten die ersten Studien zur Stadtplanung an der Universität Wien. Ein neuerlicher Aufschwung setzte schließlich 1931 ein, als Hassinger zum Ordinarius für Kulturgeographie berufen und die Südostdeutsche Forschungsgemeinschaft (SODFG) gegründet wurde. Gleich in seiner Antrittsvorlesung bekräftigte Hassinger, dass der Staat die Kulturlandschaft ,durch Landesplanung zielbewusst formen sollte" (Hassinger 1932: 187). Als Beispiele führte er den Bau von Schutzanlagen gegen Naturkatastrophen und die Neugestaltung des öffentlichen Verkehrs an.

Den endgültigen Impuls raumplanerisch zu forschen brachte schließlich die SODFG mit sich. Sie wurde im Herbst 1931 von deutschnationalen Wissenschaftlern gegründet, die sich die ,Deutschtumsforschung' in den österreichischen Grenzregionen und in den anschließenden ost- und südosteuropäischen Staaten zum Ziel gesetzt hatten. Bald folgten auch andere Volksdeutsche Forschungsgemeinschaften, die vor allem in Deutschland beheimatet waren. Die SODFG wurde in den Anfangsjahren von Hassinger geleitet. Der Vorstand bestand außerdem aus dem Historiker Hans Hirsch (1878-1940), dem Volkskundler Viktor Geramb (1884-1958) und dem Geographen Fritz Machatschek (1876-1957), der von 1928 bis 1934 die Professur für Physiogeographie an der Universität Wien innehatte. Zu den ersten Arbeiten zählten unter anderem der Aufbau einer Bibliothek über Südosteuropa und das Erstellen diverser Listen über Wissenschaftler und Bücher aus dem Südosten. Zudem wurden unzählige Projekte ins Leben gerufen, welche den deutschen Volks- und Kulturboden näher erforschen sollten. (vgl. Fahlbusch 1999)

Vom deutschen Volks- und Kulturboden sprach man bereits Ende des 19. Jahrhunderts. Doch erst die Studie des Berliner Geographen Albrecht Penck (1858-1945) ließ sie ab 1925 zu einer wissenschaftlichen und politischen Theorie werden, die zu einer der Grundlagen für Hitlers rassenbiologistischer ,Lebensraumpolitik` avancierte. „Wo deutsches Volk siedelt, ist deutscher Volksboden, da hört man deutsche Sprache und sieht deutsche Arbeit", so Penck; doch ist der deutsche Volksboden von einem „deutschen Kulturboden begleitet, der sich von dem der benachbarten Kulturgebiete unterscheidet" (Penck 1925: 62, 64) und einst von Deutschen bewohnt wurde. Mit dieser Theorie konnten nun Gebiete eingefordert werden, die nahe der deutschen Sprachinseln in Ost- und Südosteuropa gelegen waren. Der Text und die angefertigte Karte dienen wohl der nationalen Propaganda. Doch wollte Penck damals sicher keine Grundlagentheorie für den NS-Völkermord und für eine rigorose Umsiedlungspolitik schaffen. (vgl. Schultz 2018)

Die wichtigste raumplanerische Studie der SODFG, an der Mitarbeiter des Geographischen Institutes beteiligt waren, stellte der Burgenlandatlas dar. Als Vorbild dienten wohl ähnliche Unternehmungen im Deutschen Reich, wie zum Beispiel der Saar-Atlas (1934) und der Rhein-Mainische Atlas (1929). Diese entstanden nicht nur im Kontext zunehmender raumplanerischer Forschungen, sondern aufgrund ihrer grenznahen Lage zu Frankreich. Vor allem mit dem Saar-Atlas wollten Wissenschaftler und Politiker die Stellung des damals strittigen grenznahen Gebietes zum deutschen Sprachraum aufzeigen (vgl. Freund 2017). Hassinger oblag zusammen mit dem niederösterreichischen Geographen Fritz Bodo (1893-1978) die Leitung. Hassingers Assistenten Egon Lendl (1906-1989) und Walter Strzygowski (1908-1970) waren zusammen mit vielen anderen Wissenschaftlern unterschiedlicher Institute und Institutionen an der Erstellung der Karten und Begleittexte beteiligt. Dieser Atlas, der vor allem für Schulen und für die Landesplanung konzipiert wurde, sollte ohne Zweifel „der gesamtdeutschen Sache dienen" (Hassinger 1941: Vorwort). Mit ihm bauten die Herausgeber bereits vor 1938 eine intensive Verbindung mit dem nationalsozialistischen Deutschen Reich auf, da die Finanzierung weitgehend durch deutsche Reichsbehörden in Berlin erfolgte. Zudem waren einige Mitarbeiter vor 1938 Mitglied der NSDAP, wie zum Beispiel Bodo, Lendl und Strzygowski. Aus diesem Grund stellte die burgenländische Landesregierung ab 1935 kaum mehr personelle und finanzielle Ressourcen zur Verfügung. Aber auch ethnopolitische Ziele im Sinne der NS,Lebensraumpolitik' können aus den geheimen Tätigkeitsbereichen der SODFG herausgelesen werden, denn man wollte „auftauchenden ungarischen revisionistischen Bestrebungen" (PAAA, R 60291) entgegenwirken. Der Atlas erschien daher erst 1941. Eine Verbindung zur NS-Politik war also im Fachbereich Geographie bereits vor 1938 vorhanden. Im Zuge der zunehmenden politischen Verflechtung ist auch zu erkennen, dass die Kartenthemen und ihre methodische Umsetzung Veränderungen erfahren hatten. Die bei 
der Kartenherstellung angewandte Methodik ist ohne Zweifel viel komplexer geworden. So verwendete zum Beispiel Fritz Bodo für seine Karte zur Volkstumsverteilung zum ersten Mal in Österreich Sten de Geers Kugelmethode. Viele Karten enthalten im Gegensatz $\mathrm{zu}$ früheren österreichischen Atlasprojekten zudem eine große Zahl an geometrischen und sprechenden Zeichen. Lendls Karten „Burgenland im südostdeutschen Volks- und Staatsraum“ und „Burgenland im südostdeutschen Stammes- und Mundartenraum“" zeigen den deutschen Volks- und Kulturboden. Der Wiener Anthropologe Viktor Lebzelter (1889-1936) eruierte für seine anthropologischen Karten die rassenmäßige Zusammensetzung der burgenländischen Bevölkerung, indem er 5000 Burgenländerinnen und Burgenländer in 32 Gemeinden untersuchte. Solche Forschungen waren damals populär, da man damit die arische Bevölkerung gegenüber den anderen Rassen ermitteln konnte. (vgl. Svatek 2010a, 2017; Fuchs 2003)

Auch Egon Lendl, der 1931 zum „Deutschtum in der Ilovasenke" promovierte, stellte sich in den Dienst der Sache, indem er für die SODFG deutsche Sprachinseln in Südosteuropa erforschte. Seine Studie zu den ,jungen deutschen Sprachinseln im westlichen Slawonien und den benachbarten Teilen Kroatiens" bietet einen Überblick über die deutsche Besiedlungsgeschichte und zeigt dabei unter anderem auf, welche Veränderungen die Kultur- und Naturlandschaft infolge der deutschen Kolonisation erfahren hatte. Er analysierte die wirtschaftlichen Leistungen der deutschen Kolonisten und zeigte auf, dass von ihnen der Anstoß zur Landschaftsveränderung gekommen war. Die Deutschen hätten zudem neue Kulturpflanzen und neue Siedlungs- und Gehöftsformen etabliert. (vgl. Lendl 1935). Auch im Deutschen Reich führten Geographen bereits vor dem Zweiten Weltkrieg Studien zum Deutschtum grenznaher Gebiete und außerhalb Deutschlands durch. Denkt man doch nur an die raumwissenschaftlichen Untersuchungen an der Ostgrenze des Reiches von Oskar Ritter von Niedermayer (1885-1948) oder an die landeskundlichen Forschungen über Ostpommern von Hermann Lautensach (1886-1971).

Abseits der SODFG setzte sich Walter Strzygowski mit Landesplanungsthemen auseinander. Strzygowski promovierte 1931 über „Morphologische Untersuchungen im Einzugsgebiet der steirischen Salza", wurde danach aber immer mehr zum Kulturgeographen. Vor allem ab 1936 erweiterte sich sein Themenspektrum beträchtlich, indem er sich unter anderem mit Erholungsplanung und Verkehrsgeographie beschäftigte. Im Jahr 1936 betrachtete Strzygowski die Schneeverhältnisse und Geländeformen österreichsicher Skigebiete (vgl. Strzygowski 1936).
Ein Jahr später, 1937, stellte er seine „Pläne für einen Straßentunnel durch die Zentralalpen"vor, den er ungefähr zwischen Mittersill und Matrei in Osttirol verortete. Damit nahm er an der damaligen Diskussion über eine neue Straßenverbindung teil, die München schneller mit Rom verbinden sollte. (vgl. Strzygowski 1937) Eine richtige politische Relevanz scheinen seine Forschungen aber nicht gehabt zu haben. Auch erscheinen sie im Gegensatz zur Zeit nach 1938 noch etwas dürftig, da keine umfangreiche Pläne gezeichnet und planungsrelevante Umsetzungsvorschläge im Detail vorgelegt wurden.

\section{Kontinuitäten und Wandlungen ab 1938}

Ab 1938 kam es schließlich zu einer Intensivierung von Tendenzen, die bereits zuvor vorhanden waren, aber auch zu neuen Verbindungen zur Politik und zu neuen Forschungsthemen. Generell ist eindeutig eine Zunahme an raumorientierten Themen feststellbar. Im Herbst 1938 erfolgte, gemäß der Zielsetzung der RAG auch an der Universität Wien, die Gründung einer Arbeitsgemeinschaft für Raumforschung, die fortan von Hassinger geleitet und zusammen mit der Hochschule für Welthandel, der Hochschule für Bodenkunde, der Tierärztlichen Hochschulen, und der Technischen Hochschule zur Arbeitsgemeinschaft für Raumforschung an den Wiener Hochschulen ausgebaut wurde. Im Rahmen dieser Arbeitsgemeinschaft bearbeiteten einige Wissenschaftler Projekte, die sich mit der Planung des deutschen ,Lebensraumes' auseinandergesetzt hatten. Neben den Geographen Hassinger, Lendl und Strzygowski stellten sich unter anderem auch Hermann Kaserer (1877-1955) und Walter Kubiena (1897-1970) von der Hochschule für Bodenkultur, Hans Neumayer (1887-1945) vom Botanischen Institut und Erwin Ilz (1891-1954) von der Technischen Hochschule in den Dienst der Sache.

Wissenschaftliche Projekte für die Planung des deutschen ,Lebensraumes', sowohl innerhalb des Deutschen Reiches als auch in West-, Ost- und Südosteuropa, wurden nicht nur von den Wiener Geographen durchgeführt, denn auch viele Geographen des so genannten Altreiches stellten sich in den Dienst der Sache und lieferten den Nationalsozialisten im Auftrag oder durch eigene Initiativen wichtige Forschungsergebnisse und Argumente für deren ,Lebensraumpolitik'. Die Geographie war also als Raumwissenschaft für diese Politik ausgesprochen fruchtbar. Eine enge Verzahnung zwischen Geographie und Politik ergab sich nach 1938 zum Beispiel im Rahmen des von Norbert Krebs (1876-1947) herausgegebenen „Atlas des deutschen Lebensraumes" und seinen Forschungen 
zur Industrie im Warthe-Netze-Gebiet. Fritz Klute (1885-1952) forschte zur Landflucht und zur Landwirtschaft in Hessen und Kurt Brüning (1897-1961) zur Wirtschafts- und Bevölkerungsgeographie in Nordrhein-Westfalen. Zudem gab Brüning den „Atlas Niedersachsen" heraus. Friedrich Metz (1890-1969) führte wirtschaftsgeographische Untersuchungen in den schweizerischen und elsässischen Grenzgebieten durch. Diese Liste könnte noch mit weiteren Beispielen weiter fortgeführt werden (eine Liste mit einigen Forschungen der RAG vgl. Gutberger 2017).

Eine Neuerung betraf am Geographischen Institut der Universität Wien ab 1938 die Zusammenarbeit mit praxisorientierten landesplanerischen Behörden. Hassinger, Strzygowski und Bodo nahmen bereits am 13. Mai 1938 an einer Besprechung zu einer neuen Kreiseinteilung der Ostmark teil. Der Auftrag kam von der Planungsbehörde bei der Reichsstatthalterei in Wien. Anwesend waren unter anderem auch Franz Winter (1877-1956) vom BEV und Adalbert Klaar (1900-1981) und Andreas Tröster von der Wiener Planungsbehörde (OeStA, AdR ZNsZ RStH Wien Z-RO 299). Auch in den Folgejahren stand zumindest Hassinger immer wieder mit der Wiener Planungsbehörde in Verbindung. Ein Briefkontakt kam unter anderem mit Adalbert Klaar zustande, der Hassinger über den Fortschritt bei der Erstellung diverser Planungskarten (Baualterpläne, Bevölkerungsdichtekarten, Industrie- und Gewerbekarten) unterrichtete (OeStA, AdR ZNsZ RStH Wien Z-RO 303). Für die Planungsbehörden in Oberund Niederdonau verfasste Hassinger physio- und kulturgeographische Ortsbeschreibungen von ausgewählten Gemeinden, wie zum Beispiel Liebenau und Fels am Wagram. Mit diesen Beschreibungen sollte festgestellt werden, ob diese Auswahlgemeinden für Be- und Aussiedlungen in Frage kommen würden. (UAW, NL Hassinger Kt. 14; UAW, NL Hassinger Kt. 16) Hassingers Expertenwissen war auch bei diversen Sitzungen von Planungsbehörden gefragt. Als zwei Beispiele sollen hier die Besprechung zur wirtschaftlichen Entwicklung des Rauristales und der Vegetationskartierung von Salzburg genannt werden (UAW, NL Hassinger Kt. 1). Im Jahr 1938 erhielt Hassinger zudem vom Wiener Bürgermeister Hermann Neubacher (1893-1960) einen Auftrag, das Wiener Donaugelände im Hinblick auf eine zukünftige Bebauung zu prüfen. Wien sollte wie viele andere Städte des Deutschen Reiches um- und neugestaltet werden, wobei in Wien vor allem die von vielen Juden bewohnte Leopoldstadt ins Auge gefasst wurde. Hassingers Gutachten setzte sich mit neuen Verkehrsverbindungen ebenso auseinander wie mit dem Ausbau des Hafens und neuer Industrieanlagen (UAW, NL Hassinger Kt. 15). Auch Strzygowski stellte sich in den Dienst dieser Forschungen. Doch wurde er nicht von Neubacher beauftragt, sondern handelte aus eigener Initiative. Neue Fernstraßen, Bahnhöfe, Siedlungs- und Industriegebiete waren in seiner Studie ebenso Thema wie eine umfassende Planung des zweiten Bezirkes (vgl. Strzygowski 1939).

Auch die anderen von Strzygowski verfassten landesplanerischen Studien waren nicht direkt von politischen Behörden initiiert worden. Doch steht die politische Relevanz seiner Forschungen heute außer Zweifel. Sein 1938 geschriebener Artikel „Die Bedeutung des Skisportes für Österreich in Gegenwart und Zukunft" sollte wohl unter anderem alle Deutschen motivieren, Österreich als Zentrum des Wintersporttourismus anzuerkennen. Im selben Jahr verfasste er zudem einen Artikel zur österreichischen Landesplanung, worin er für einen umfassenden Landesflächenwidmungsplan plädierte. Strzygowski setzte sich aber auch kritisch mit der Bevölkerungsentwicklung und einer möglichen besseren Erschließung des Alpenraumes auseinander. (vgl. Strzygowski 1938b) Seine Habilitationsschrift des Jahres 1940, die heute als verschollen gilt, beschäftigte sich mit einer möglichen künftigen Raumordnung Österreichs. Die Habilitationsgutachten von Hassinger und Johann Sölch geben uns Auskunft darüber, dass Strzygowskis Arbeit wohl viele verschiedene Aspekte der Stadt- und Landesplanung aufgriff, von der damals durchaus schon populären ,Zentrale Orte'-Theorie über die Umsiedlung deutscher Volksgruppen bis hin zu einer Optimierung der rassischen Bevölkerungsstruktur (UAW, PA Strzygowski 3566).

Strzygowski war ohne Zweifel derjenige Geograph, der sich am meisten dem NS-Sprachgebrauch und der antisemitischen Politik angepasst hatte. Er trat 1938 für die „Reinigung Wiens von der Judenplage“ ein (Strzygowski 1938: 423), und 1939 konstatierte er, dass Wien „von Juden und Verrätern beherrscht wurde“, sodass „Wien im eigenen Land verhasst" war. „Seit der Tat des Führers liegt das alles wie ein Spuk hinter uns" (Strzygowski 1939: 42). In seinem 1942 publizierten Artikel „Erholungsräume und Reiseziele der Bevölkerung Wiens" kritisierte er ,eine starke Verseuchung mit Juden" mancher Sommerurlaubsorte (Strzygowski 1942: 327).

Der Gauatlas Niederdonau war das umfangreichste raumplanerische Projekt während der NS-Zeit. Im Jahr 1940 vom niederösterreichischen Geographen Fritz Bodo initiiert und zum großen Teil von der Gauverwaltung Niederdonaus finanziert, sollte er der Raumforschung im Sinne der Politik des Dritten Reiches dienen. Hassinger wurde neben Bodo zum zweiten Leiter des Atlasvorhabens bestellt. Die über 20 Mitarbeiter kamen aus den unterschiedlichsten Universitätsinstituten (Geographie, Germanistik, 
Geschichtswissenschaft, Archäologie, Volkskunde usw.) und der Gauverwaltung Niederdonaus, die mit der SODFG, der Südosteuropagesellschaft, dem Statistischen Amt für die Donau- und Alpengaue, der Sudetendeutschen Anstalt für Heimatforschung in Reichenberg und diversen Planungsbehörden kooperierten. Zusätzlich wurden von der RAG einige Projekte finanziert, die als Vorarbeiten zu Karten angesehen werden können. Unter diesen befanden sich zum Beispiel Studien zu den Wüstungen Niederdonaus, über die anthropologischen Merkmale und den Altersaufbau der Bevölkerung und die Entwicklung der Einwohnerzahl. Diese wurden vor allem vom Geographischen Institut mit diversen Mitarbeitern durchgeführt. Bei der Erforschung der Wüstungen kooperierte Hassinger zum Beispiel mit der Landesbauernschaft Donauland, mit Heinrich Kunnert (1904-1979) vom Archiv in Eisenstadt, mit dem Obersulzer Gemeindearzt Heinrich Weigel und mit dem Brünner Lehrer Hans Reutter, die ihm von ihren jeweiligen Heimatgebieten Daten zur Verfügung stellten. Diese waren nicht nur für den Gauatlas von Bedeutung, sondern sollten auch für mögliche Neubesiedlungen zur Verfügung gestellt werden. (Svatek 2010 a, 2015)

Eine Kontinuität zur Zeit vor 1938 stellen die ethnographischen Forschungen Wiener Geographen in Südosteuropa dar. Allerdings erfolgten diese unter anderen politischen Rahmenbedingungen, da ab dieser Zeit die Raumeroberung im Kontext der ,völkischen' Neuordnung Europas im Vordergrund stand. Dabei leisteten die Geographen auch einen Beitrag zum ,völkischen' Neuordnungskonzept Europas, „das mittels Vertreibungen, Deportationen und Völkermord Siedlungsgebiete für arische Deutsche schaffen sollte" (Wildt 2016: 38). Während die Studie zur Umsiedlung der Südtiroler, die Hassinger zusammen mit Strzygowski und dem Geographen Hans Graul (1909-1997) 1939 ausgearbeitet hatte, noch vom Verein für das Deutschtum im Ausland im Auftrag gegeben wurde, handelte vor allem Hassinger in den Folgemonaten aus eigener Initiative. Vor allem die beiden Projekte über die „Neuordnung der deutschen Volksgruppengebiete im innerkarpatischen Raum“ und die „Bestandsaufnahme der deutschen Volksgruppen im mittleren Donauraum und geographische Untersuchung ihrer Siedlungsgebiete" sollten mögliche Umsiedlungsfragen aufzeigen und der Politik zur Verfügung gestellt werden, „wenn eine dieser Siedlungsfragen politische angeschnitten werden sollte" (UAW, Nachlass Hassinger Kt. 16). Hassinger verwendete für diese, völkische' Umsiedlungsplanung den Begriff „Flurbereinigung“ (UAW, Nachlass Hassinger Kt. 26), der auch von Hitler in seiner Reichstagsrede vom 6. Oktober 1939, im Zuge seiner Ausführungen zur völkischen Neuordnung Europas, ausgesprochen wurde (Wildt 2016: 37). Daher hat auch im Bereich der Geographie die vom Historiker Mitchell G. Ash aufgestellte These seine Gültigkeit, dass Wissenschaftler während der NS-Zeit nicht nur als „Opfer" der Politik, sondern als „bewusst handelnde Subjekte“ (Ash 2002: 32-33) gelten. Vielmehr lassen sich auf Gegenseitigkeit aufgebaute Beziehungen feststellen, so zum Beispiel Hassingers Kontakt zur Forschungsstaffel z.b.V.; 1943 vom Oberkommando der Wehrmacht gegründet führte sie natur- und kulturwissenschaftlich Forschungen in wenig erschlossenen Gebieten Ost- und Südosteuropas durch (vgl. Flachowsky 2011). Hassinger sandte ihr von sich aus diverse landeskundliche Bücher und Karten über Südosteuropa und den im Rahmen der Arbeitsgemeinschaft für Raumforschung von Alois Jaschke erarbeiteten Atlas von Kroatien. Als Gegenleistung erhielt Hassinger die Möglichkeit, ab 1944 in der Arbeitsgemeinschaft für wehrwissenschaftliche Geländeforschung und Wehrgeologie mitzuarbeiten (vgl. Svatek 2010 b).

\section{Resümee}

Eine Verbindung zur Politik besteht in der Geographie und Kartographie bereits seit der Antike. So haben Kriege die geographische Erforschung der eroberten Gebiete nach sich gezogen oder auch das Fehlen von geeignetem Kartenmaterial aufgezeigt. Als ab dem 19. Jahrhundert an deutschsprachigen Universitäten geographische Lehrstühle und Institute gegründet wurden, haben Geographen immer wieder mit Expertisen gewollt oder ungewollt der Politik zugearbeitet oder Aufträge von politischen Behörden erhalten. Dadurch kann der Geographie als Wissenschaft auf keinem Fall eine Wertfreiheit attestiert werden. Auch eine Verbindung zur völkisch-nationalistischen Ideologie war am Geographischen Institut der Universität Wien bereits am Beginn des 20. Jahrhunderts vorhanden (vgl. Henniges 2015). Mit der NS-Herrschaft änderte sich nun die „Radikalität der Politisierung" (Brogiato 2005: 68). Ansätze und Themen der Geographen (Deutschtum, politische Geographie usw.) passten hervorragend zu nationalsozialistischen Zielsetzungen, wie zum Beispiel der Schaffung eines reinrassigen Herrenvolkes und der Expansionspolitik. „Die stärksten Verstrickungen von geographischer Wissenschaft und Politik im Nationalsozialismus entstanden in der anwendungsorientierten Raumforschung. Hier verbanden sich tradierte Forschungsfelder der Volks- und Kulturbodenforschung, neue Ansätze funktionsräumlicher Theorien und Modelle mit der nationalsozialistischen Blut-und-Boden-Ideologie." (Brogiato 2005: 68) 
Auch die Wiener Geographen haben ihren Beitrag dazu geleistet. Geographische Projekte wurden im Rahmen der SODFG bereits vor 1938 vom NSDeutschland finanziert. Nach dem Anschluss Österreichs an das Deutsche Reich 1938, führte man die bereits zuvor getätigten, völkischen' Studien fort. Eine Neuerung betraf vor allem die Zusammenarbeit des Geographischen Instituts mit der praxisorientierten Stadt- und Landesplanung. Die Verbindung zu politischen Behörden wurde intensiviert. Ein Zuarbeiten an die NS-Eroberungs- und ,Lebensraumpolitik' war explizit beabsichtigt.

Nach dem Zweiten Weltkrieg konnte Hassinger seine Karriere ungehindert fortsetzen, da er kein Mitglied der NSDAP war. Unter anderen politischen Rahmenbedingungen verfolgte er weiterhin eine enge Verbindung zu politischen Institutionen, mit denen er vor allem in der 1946 von ihm mitgegründeten Kommission für Raumforschung und Wiederaufbau der Österreichischen Akademie der Wissenschaften kooperierte. In dieser Kommission wurde unter anderem der Niederösterreichatlas erarbeitet, der auf Vorarbeiten zum Gauatlas Niederdonau aufbauen konnte. (Svatek 2015)

Das hier vorgestellte Thema eignet sich im Schulunterricht dazu, die komplexen Beziehungen zwischen Geographie und Politik in Vergangenheit und Gegenwart zu erörtern, insbesondere die Vernetzung von Wissenschaftlern, politischen Entscheidungsträgern und Politikern in einer Diktatur. Eine profunde Kenntnis über die ,Lebensraumpolitik‘ im Nationalsozialismus kann auch vergleichend zur Diskussion über aktuelle gesellschaftliche Ideologien verschiedener Staaten und Herrschaftsformen herangezogen werden. Denn „Unterricht in Geographie setzt(e) sich traditionell mit Staaten, Bevölkerung (Gesellschaft) und Wirtschaft auseinander - Bereiche, die auch politische Dimensionen beinhalten können" (Sitte 2014: 29). Gleichzeitig würde sich eine Auseinandersetzung mit der Geschichte des eigenen Faches im Nationalsozialismus auch dazu eignen, regionale Konflikte besser einschätzen und demokratisches Denken weiterentwickeln zu können. Zudem ist dieses Thema zum fächerübergreifenden Unterrichten prädestiniert, da sich auch andere Unterrichtsfächer (Geschichte, Germanistik) mit Politik, Gesellschaft und Raum in der Gegenwart und Vergangenheit auseinandersetzen.

\section{Acknowledgement}

Der Artikel wurde im Zuge des Projektes „Wissenschaft und Politik am Geographischen Institut der Universität Wien während der NS-Zeit" beim Zukunftsfonds der Republik Österreich verfasst.

\section{Literatur und Quellen}

Ash, M. G. (2002): Wissenschaft und Politik als Ressourcen für einander. In: Bruch, R., Kaderas, B. (Hrsg.), Wissenschaften und Wissenschaftspolitik. Bestandsaufnahmen zu Formationen, Brüchen und Kontinuitäten. S. 32-51.

Baas, K. (2015): „Erdkunde als politische Angelegenheit". Geographische Forschung und Lehre an der Universität Münster zwischen Wissenschaft und Politik (1909-1958). Aschendorff Verlag, Münster.

Böhm, H. (2008): Geographie. In: Elvert, J., NielsenSikora, J. (Hrsg.), Kulturwissenschaften und Nationalsozialismus. S. 359-389.

Brogiato, H.-P. (2005): Geschichte der deutschen Geographie im 19. und 20. Jahrhundert - ein Abriss. In: Schenk, W., Schliephake, K. (Hrsg.), Allgemeine Anthropogeographie. S. 41-81.

Dörries, H. (1937): Geographie als Grundlage von Raumforschung und Landesplanung. In: Verhandlungen und wissenschaftliche Abhandlungen des 26. Deutschen Geographentages zu Jena. S. 41-53.

Fahlbusch, M. (1999): Wissenschaft im Dienste der nationalsozialistischen Politik? Die „Volksdeutschen Forschungsgemeinschaften" von 1931-1945. Nomos, Baden-Baden.

Flachowsky, S. (2011): „Die Forschungsgruppe SchulzKampfhenkel steht jetzt für Ostaufgaben zur Verfügung“. Otto Schultz-Kampfhenkel als Beauftragter für Sonderaufgaben der erdkundlichen Forschung. In: Flachowsky, S., Stoecker H. (Hrsg.), Vom Amazonas an die Ostfront. Der Expeditionsreisende und Geograph Otto Schulz-Kampfhenkel (1910-1989). S. 240-302.

Freund, W. (2017): Saar-Atlas. In: Fahlbusch, M., Haar, I., Pinwinkler, A. (Hrsg.), Handbuch der völkischen Wissenschaften. Forschungskonzepte - Institutionen - Organisationen - Zeitschriften. S. 2125-2129.

Fuchs, B. (2003): „Rasse“, „Volk“, „Geschlecht“: anthropologische Diskurse in Österreich 1850-1960. Campus, Frankfurt am Main-New York.

Geisler, W. (1942): Kartographie und Raumforschung. Vorschläge zum Ausbau einer Methodik von Planungskarten. In: Raumforschung und Raumordnung VI. S. 41-50.

Hassinger, H. (1910): Über Aufgaben der Städtekunde. In: Petermanns Mitteilungen 56. S. 289-294.

Gutberger H.-J. (2017): Raumentwicklung, Bevölkerung und soziale Integration: Forschung für Raumplanung und Raumordnungspolitik 1930-1960. Springer, Wiesbaden.

Hassinger, H. (1910): Beiträge zur Siedlungs- und Verkehrsgeographie von Wien. In: Mitteilungen der k. k. Geographischen Gesellschaft in Wien 53. S. 5-88.

Hassinger, H. (1912): Wiener Heimatschutz- und Verkehrsfragen. Freytag \& Berndt, Wien.

Hassinger, H. (1916): Kunsthistorischer Atlas der k. k. Reichshaupt- und Residenzstadt Wien und Verzeichnis der erhaltenswerten historischen Kunst- und Naturdenk- 
male des Wiener Stadtbildes. Österreichische Kunsttopographie XV. Schroll, Wien.

Hassinger, H. (1932): Der Staat als Landschaftsgestalter. In: Zeitschrift für Geopolitik IX. S. 117-122, 182-187.

Hassinger, H. (1941): Vorwort. In: Hugo Hassinger (Hrsg.), Burgenland. Ein deutsches Grenzland im Südosten.

Leendertz, A. (2017): Reichsarbeitsgemeinschaft für Raumforschung. In: Fahlbusch, M., Haar, I., Pinwinkler, A. (Hrsg.), Handbuch der völkischen Wissenschaften. Forschungskonzepte - Institutionen - Organisationen Zeitschriften. S. 1926-1934.

Lendl, E. (1935): Die jungen deutschen Sprachinseln im westlichen Slawonien und den benachbarten Teilen Kroatiens. In: Geographischer Jahresbericht aus Österreich XVIII. S. 79-95.

Meyer, K. (1937a): Raumforschung und Geographie. In: Verhandlungen und wissenschaftliche Abhandlungen des 26. Deutschen Geographentages zu Jena. S. 34-40.

Meyer, K. (1937 b): Raumforschung. In: Raumforschung und Raumordnung 1. S. 2-3.

Henniges, N. (2015): „Naturgesetze der Kultur“: Die Wiener Geographen und die Ursprünge der „Volks- und Kulturbodentheorie“. In: ACME. An International EJournal for Critical Geographies 14/4. S. 1309-1351.

OeStA (Österreichisches Staatsarchiv), AdR ZNsZ RStH Wien Z-RO 299: Ergebnis der Besprechung vom 13.5. 1938 bezüglich der von uns vorgeschlagenen Kreiseinteilung im Gebiete Österreich.

OeStA, AdR ZNsZ RStH Wien Z-RO 303: Briefverkehr Hugo Hassinger mit Adalbert Klaar.

PAAA (Politisches Archiv Auswärtiges Amt Berlin), R 60291: Tätigkeitsbericht der Südostdeutschen Forschungsgemeinschaft über das Rechnungsjahr 1934/35.

Penck A. (1925): Deutscher Volks- und Kulturboden. In: Loesch, K. (Hrsg.), Volk unter Völkern. Bücher des Deutschtums 1. S. 62-73.

Rössler, M. (1987): Die Institutionalisierung einer neuen Wissenschaft im Nationalsozialismus: Raumforschung und Raumordnung 1935-1945. In: Geographische Zeitschrift 75. S. 177-194.

Schmitz-Berning, C. (2007): Vokabular des Nationalsozialismus. Walter de Gruyter, Berlin.

Schultz, H-D (2010): Was „ist“ Geographie? Was „ist“ sie nicht? Zur Konfiguration des Faches als politisch relevante „reine“ (Natur-)Wissenschaft. In: Tenorth, H-E. (Hrsg.), Geschichte der Universität Unter den Linden 1810-2010. Band 5: Transformation der Wissensordnung. S. 651-674.

Schultz, H-D (2018): Albrecht Penck: Vorbereiter und Wegbereiter der NS-Lebensraumpolitik? In: E\&G Quaternary Sci. J., 66. S. 115-129.

Sitte, C. (2014): Politische Bildung im Geographieunterricht. In: Geographie aktuell \& Schule 208/36. S. 29-34.

Strzygowski, W. (1936): Skigeographie von Österreich. In: Skileben in Österreich. Jahrbuch des österreichischen Skiverbandes. S. 27-38.
Strzygowski, W. (1937): Pläne für einen Straßentunnel durch die Zentralalpen. In: Mitteilungen der Geographischen Gesellschaft in Wien 80. S. 93-95.

Strzygowski, W. (1938a): Die Bedeutung des Skisportes für Österreich in Gegenwart und Zukunft. In: Skileben in Österreich. Jahrbuch des österreichischen Skiverbandes. S. 79-99.

Strzygowski, W. (1938b): Die geographischen Grundlagen einer Landesplanung in Österreich. In: Raumforschung und Raumordnung 2/9. S. 418-425.

Strzygowski, W. (1939): Vorschläge für die künftige Gestaltung Wiens. In: Nachrichtenblatt des Vereins für Geschichte der Stadt Wien 1/3. S. 41-64.

Strzygowski, W. (1942): Erholungsräume und Reiseziele der Bevölkerung Wiens. In: Mitteilungen der Geographischen Gesellschaft in Wien 85. S. 324-327.

Svatek,P.(2010a):FritzBodo-Atlaskartographieinden 1930er und frühen $1940 \mathrm{er}$ Jahren. In: Mitteilungen der Österreichischen Geographischen Gesellschaft 152. S. 323-338.

Svatek,P.(2010 b):HugoHassingerundSüdosteuropa.Raumwissenschaftliche Forschungen in Wien (1931-1945). In: Sachse C. (Hrsg.), „Mitteleuropa“ und „Südosteuropa" als Planungsraum. Wirtschafts- und kulturpolitische Expertisen im Zeitalter der Weltkriege. S. 290-311.

Svatek, P. (2015): Raumforschung an der Universität Wien im 20. Jahrhundert. Kontinuitäten und Wandlungen einer multidisziplinären und politisch orientierten Forschungsrichtung. In: Kniefacz, K., Nemeth, E., Posch, H., / Stadler, F. (Hrsg.), Universität - Forschung Lehre. Themen und Perspektiven im langen 20. Jahrhundert. 650 Jahre Universität Wien - Aufbruch ins neue Jahrhundert, Bd. 1. S. 241-259.

Svatek, P. (2017): Burgenlandatlas. In: Fahlbusch, M., Haar, I., Pinwinkler, A. (Hrsg.), Handbuch der völkischen Wissenschaften. Forschungskonzepte - Institutionen - Organisationen - Zeitschriften. S. 2057-2060.

UAW (Universitätsarchiv Wien), Nachlass Hassinger Kt. 1: diverse Briefe und Besprechungsprotokolle.

UAW, Nachlass Hassinger Kt. 14: Briefe Hassingers an den Reichsstatthalter in Oberdonau als Planungsbehörde.

UAW, Nachlass Hassinger Kt. 15: Vorschlag zur Planung des Wiener Donaugeländes im Rahmen der Gesamtgestaltung des Wiener Verkehrsnetzes vom Juli 1938.

UAW, Nachlass Hassinger Kt. 16: Projektansuchen „Entwurf von geographischen Ortsbeschreibungen für die Wunschbilder der Planungsbehörden Wien, Niederdonau und Oberdonau und Beschreibung von politischen Bezirken und Naturlandschaften".

UAW, Nachlass Hassinger Kt. 16: Projektansuchen „Neuordnung der deutschen Volksgruppengebiete im innerkarpatischen Raum“ und „Bestandsaufnahme der deutschen Volksgruppen im mittleren Donauraum und geographische Untersuchung ihrer Siedlungsgebiete“.

UAW, Nachlass Hassinger Kt. 26: Vortrag Hassingers auf Tagung der deutschen wissenschaftlichen Ost- und Südostinstituten in Breslau, 25.-27.11.1941. 
UAW, Nachlass Hassinger Kt. 27: Bericht „Raumforschung und Raumordnung in Österreich“.

UAW, PA Strzygowski 3566: Habilitationsgutachten.

Wardenga, U. (1995): Geschichtsschreibung in der Geographie. In: Geographische Rundschau 47. S. 523-525.

Wardenga, U. (2006): Geographie. In: Eckart, W., Sellin, V., Wolgast, E. (Hrsg.), Die Universität Heidelberg im Nationalsozialismus. S. 1213-1244.
Wardenga, U., Henniges, N., Brogiato, H. \& B. Schelhaas (2011): Der Verband deutscher Berufsgeographen 1950-1979. Eine sozialgeschichtliche Studie zur Frühphase des DVAG. forum ifl 16. Leibniz Institut für Länderkunde, Leipzig.

Wildt, M. (2016): Völkische Neuordnung Europas. In: Hohls, R., Kaelble, H. (Hrsg.), Geschichte der europäischen Integration bis 1989. S. 37-44. 